\title{
Electroconvulsive therapy in single manic episodes: a case series
}

\author{
H Mohammadbeigi', S Alizadegan'2, M Barekatain' \\ 'Department of Psychiatry, Isfahan University of Medical Sciences, Isfahan, Iran \\ 2Department of Epidemiology, Reproductive Medicine Research Center, Royan Institute, Isfahan, Iran
}

\begin{abstract}
Objective: To evaluate the effectiveness of electroconvulsive therapy in the treatment of Bipolar I Disorder patients with a single manic episode. Method: In a retrospective study, we reviewed medical records of inpatients who had been admitted to treat a single manic episode of Bipolar I Disorder at Noor University Hospital, Isfahan, Iran between September 2004 and December 2008. Results: Out of a total of 275 single episode manic patients, 39 underwent ECT. Male/ female ratios were 19/20 (48.7\% vs. 51.3\%) for the ECT series and $137 / 99$ (58\% vs. 42\%) for the exclusive pharmacological treatment series ( $p>0.05)$. Mean age of patients in the ECT series (23.64 \pm 8.00 ) was significantly lower than the pharmacological treatment series (27.65 $\pm 11.30, \mathrm{p}=0.008)$. The mean length of stay (LOSs) for

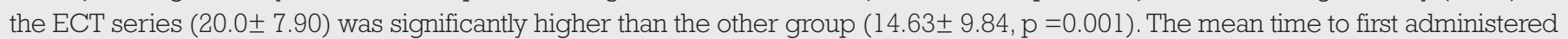
ECT (tECT) was $4.35 \pm 3.79$. There was no significant difference between the mean LOSs of the pharmacologic treatment series and the mean "LOSs minus tECT" (LOS-tECT) variable $(16.57 \pm 8.43)$ in the ECT series $(\mathrm{p}>0.05)$. The mean duration from the onset of the symptoms to time of admission was $19.22 \pm 3.53$ for the ECT series. Catatonia was the indication for application of ECT in one patient (2.6\%), while 25 (64.10\%) received ECT because of aggressive behavior. The proportion of patients administered chemical and physical restraints before ECT (77\%) significantly dropped (7.7\%) after ECT administration $(p<0.001)$. Conclusion: ECT is an effective, safe, and probably underused treatment method for single episode manic patients. Reducing the time until commencement of ECT should be considered, even in a single manic episode.
\end{abstract}

Key words: Electroconvulsive therapy; Bipolar I disorder; Single manic episode

Received: 21-08-2009

Accepted: 09-12-2009

\section{Introduction}

Introduced by Cerletti and Bini in 1938, Electroconvulsive Therapy (ECT) is considered an effective remedy in the management of severe psychiatric disorders including affective disorders. ${ }^{1-4}$ Despite the rapid progresses achieved in pharmacotherapy, electroconvulsive therapy is an effective treatment strategy in severe and treatment-resistant mental disorders. ${ }^{5}$ The effectiveness of ECT in the treatment of bipolar patients, especially patients with treatment-resistant bipolar disorder, is well documented. Response rate has been reported to be $80 \%$ in the treatment of manic episodes. ${ }^{6}$ ECT has also been found to contribute to improvement of patients with mixed bipolar episodes in cases of failure of pharmacotherapy. ${ }^{7}$ Although some studies note the fact that use of ECT in treatment of mania is associated with increased length of stay (LOS), it appears that this is due to the delay between hospitalization and administration of ECT (i.e. in most cases, ECT was only applied after a course of pharmacologic treatment failed to improve the condition of the patient). ${ }^{8}$ It has also been reported that electroconvulsive therapy is associated with improvement in symptoms and quality of life of individuals suffering from mental disorders. The positive effects of ECT are reported by both physicians and patients. ${ }^{9-10}$

Despite the effectiveness of electroconvulsive therapy and its safety, there has always been a negative attitude towards this treatment among both the public and the media. A survey conducted on a community sample reported that only $1.2 \%$ of the sample consisting of 1737 individuals was in favor of such a treatment. ${ }^{10}$ It seems that patients receiving ECT and their relatives showed a much more favorable perspective regarding such treatment. ${ }^{11-15}$ Whether the prejudicial views of the public play a role in reserving ECT as the last line treatment of mood disorders by treating physicians, remains uncertain.

Some recent studies have evaluated the effectiveness of ECT in the treatment of first-episode schizophrenia and have noted 
positive aspects such as: more significant improvement of patients (compared to the patients receiving pharmacotherapy) based on pre- and post- treatment assessment using standard scales. ${ }^{16}$

A number of studies have also assessed the effectiveness of application of ECT to patients with mania and have concluded that the use of ECT during hospitalizations for mania has been associated with longer LOS; an effect which disappeared as the LOSs minus time to first given ECT (LOS- tECT) was considered as the response variable. ${ }^{8}$ There is a paucity of evidence regarding the effectiveness of ECT for first-episode mania. Based on the practice guidelines for the treatment of patients with bipolar disorder, application of ECT for first-episode mania is reserved for cases with severe or treatment- resistant symptoms as well as for patients experiencing severe mania during pregnancy. ${ }^{1,17}$

Considering the rapid and effective response of bipolar patients to ECT as well as the limited side effect profile, it seems that there is a need to evaluate the application of this treatment earlier in the course of the disorder in order to examine whether any revisions for the existing guidelines and algorithms are necessary. In this study, we aim at reviewing the treatment course of a series of patients hospitalized in their first episode of mania.

\section{Method}

\section{Study design}

This study was conducted as a case-series based on retrospective review of the medical records of all adult in-patients admitted to the psychiatry unit of Noor University Hospital, affiliated to Isfahan University of Medical Sciences, Isfahan, Iran. The study protocol was approved by the research council of the Behavioral Sciences Research Center, Isfahan University of Medical Sciences.

\section{Subjects}

The records of patients hospitalized during the interval between September 2004 and December 2008 at Noor University Hospital were enrolled in the study. Patients were given clinician-rated diagnoses of Bipolar I Disorder, single manic episode, using the Diagnostic and Statistical Manual of Mental disorders, 4th edition (DSM-IV) ${ }^{18}$ criteria, based upon discussion within the psychiatry unit by the attending psychiatrist and the third author M.B. Indications for ECT in these patients and the number of treatment sessions had been determined by the attending psychiatrist, but in order for their data to be included in this study, all patients had to have received a minimum of $2 \mathrm{ECT}$ sessions.

\section{ECT treatment course}

In accordance with the ECT protocol of the Isfahan Province Mental Health Committee, informed consent was provided either by the patients or by one of the patient's first-degree relatives, authorizing the responsible psychiatrist to consider ECT as the treatment plan based on his/her clinical judgment.

ECT had been applied after patients' physical status was evaluated with electrocardiogram, laboratory tests, and physical examination including neurological examination. All patients were visited by a consultant anesthesiologist prior to the final decision for ECT. Wherever indicated, consultation and liaison had been provided by other medical services.

All patients received bitemporal ECT. A brief-pulse ECT device (Thymatron- DGx, Somatics, LLC, Lake Bluff, IL) was used for all subjects with a 1.0- milliseconds stimulus pulse width. A modified dose titration method was used to measure seizure threshold (ST) at the first ECT session. ${ }^{19}$ The initial dose was 50.4 mc. The seizure induction was repeated by stepwise increased stimulus doses after 30 seconds if the ECT stimulus could not elicit generalized convulsive activity lasting for at least 25 seconds objectively. The stimulus dose that induced seizure activity as recorded by EEG was defined as the ST. The stimulus level used was defined as the ST level. ECT was administered 3 times a week. The treatments were continued with fixed stimuli, ensuring that the motor seizure duration exceeded 25 seconds. If the generalized motor seizure was shorter than 25 seconds, the patient was re-stimulated after approximately 60 seconds with a 100.8-mc increased charge, but if no seizure occurred, restimulation was given after approximately 30 seconds with a same increased charge.

\section{Statistical analysis}

Independent t-test, chi-square, and McNemar test were used for statistical analysis

\section{Results}

Of a total of 275 patients diagnosed with a single manic episode, 39 patients who underwent ECT during hospitalization were closely reviewed. Male/female ratios were 19/20 (48.7\% vs. $51.3 \%$ ) for the ECT series and $137 / 99$ (58\% vs. 42\%) for the exclusive pharmacologic treatment patients ( $p>0.05)$. According to the records, 26 (66.7\%) of the ECT patients were married, 11 (28.2\%) single and 2 (5.1\%) divorced. In the pharmacotherapy group, there were 144 (61\%) married, 4 (2\%) divorced, and 88 (37\%) single patients, which was not significantly different from the ECT group. The age of the ECT patients ranged between 17 and 56 years with a mean of $23.64 \pm 8.00$ years. This figure was significantly lower than the mean of the single manic patients who exclusively received drug therapy $(27.65 \pm 11.30 ; \mathrm{p}=0.008)$. The mean level of education in the ECT group (10.89 \pm 2.44 years) was not significantly different from the mean of the pharmacotherapy group (10.28 \pm 2.68 years). Table I summarizes the socio-demographic features of the patients.

Lifelong prevalence of major psychiatric disorders in the first

\begin{tabular}{|c|c|c|c|c|}
\hline Characteristics & \multicolumn{2}{|c|}{$\begin{array}{l}\text { ECT Group } \\
\text { No patients } \\
39\end{array}$} & \multicolumn{2}{|c|}{$\begin{array}{c}\text { Pharmacotherapy group } \\
\text { No patients } \\
236\end{array}$} \\
\hline Sex & $N$ & $\%$ & $\mathrm{~N}$ & $\%$ \\
\hline $\begin{array}{l}\text { Male } \\
\text { Female }\end{array}$ & $\begin{array}{l}19 \\
20\end{array}$ & $\begin{array}{l}49 \\
51\end{array}$ & $\begin{array}{l}137 \\
99\end{array}$ & $\begin{array}{l}58 \\
42\end{array}$ \\
\hline \multirow[t]{2}{*}{$\begin{array}{l}\text { Family status } \\
\text { Married } \\
\text { Not married }\end{array}$} & $\begin{array}{l}26 \\
13\end{array}$ & $\begin{array}{l}67 \\
33\end{array}$ & $\begin{array}{l}144 \\
92\end{array}$ & $\begin{array}{l}61 \\
39\end{array}$ \\
\hline & \multicolumn{2}{|c|}{ Mean } & \multicolumn{2}{|c|}{ SD } \\
\hline $\begin{array}{l}\text { Age }^{\star} \\
\text { Education (years) }\end{array}$ & $\begin{array}{l}23.64 \\
10.89\end{array}$ & $\begin{array}{l}8.00 \\
2.44\end{array}$ & $\begin{array}{l}27.65 \\
10.28\end{array}$ & $\begin{array}{l}11.30 \\
2.68\end{array}$ \\
\hline $\begin{array}{l}\text { SD: Standard De } \\
{ }^{*} P<0.05\end{array}$ & ation & & & \\
\hline
\end{tabular}


degree relatives of probands in the ECT group was 64\%. Table II represents these features.

\begin{tabular}{|l|l|l|}
\hline \multicolumn{2}{|l|}{ Table II: Family history of patients } \\
\hline Characteristics & N & $\%$ \\
\hline FHx of schizophrenia & 3 & 7.70 \\
FHx of BID & 11 & 28.2 \\
FHX of MDD & 4 & 10.3 \\
FHx of SA & 6 & 15.4 \\
FHx of PTSD & 1 & 2.60 \\
FHx of treatment with ECT & 0 & 0 \\
\hline
\end{tabular}

FHx: Family History; BID: Bipolar I Disorder; MDD: Major Depressive Disorder; SA: Substance Abuse; PTSD: Post Traumatic Stress Disorder; ECT: Electroconvulsive Therapy

According to the records, catatonia was the main cause for application of ECT in one patient (2.6\%), while 25 (64.10\%) of patients received the treatment because of aggressive behavior (including suicidal and homicidal patterns). For the rest of the patients, ECT was considered either because of severity of other symptoms or because of lack of response to medication. Table III depicts the summary of clinical characteristics of these patients.

Table III: Clinical characteristics of patients treated with ECT

\begin{tabular}{|l|l|l|}
\hline Characteristics & N & $\%$ \\
\hline Catatonia & 1 & 2.6 \\
Irritability & 35 & 89.7 \\
Aggression & 25 & 64.1 \\
Elation & 7 & 17.9 \\
Hyper-sexuality & 7 & 17.9 \\
Free-spending behavior & 13 & 33.3 \\
Psychotic features & 36 & 92.3 \\
Hallucination & 12 & 30.8 \\
Delusion & 36 & 92.3 \\
Hyper-religiosity & 7 & 17.9 \\
Distractibility & 6 & 15.4 \\
Hyper-talkativeness & 33 & 84.6 \\
Hyperactivity & 12 & 30.8 \\
Increased energy & 8 & 20.5 \\
\hline
\end{tabular}

The mean duration of time from the onset of the symptoms to the time of admission was 19.22 3.53 days. The mean of length of stay (LOSs) for the ECT series was 20.0 7.90 days. In single manic patients who exclusively received drug therapy, the mean LOSs was $14.63 \pm 9.84$ days. Thus, patients in the ECT series have significantly longer LOSs $(p=0.001)$. The mean time to first-given ECT (tECT) was $4.35 \pm 3.79$ days. Considering LOSs minus tECT as the response variable, there was no significant difference between the mean LOSs in the pharmacologic treatment series and the mean "LOS-tECT" variable $(16.57 \pm 8.43)$ in the ECT series.

Thirty patients had received mood stabilizers, namely Valproate and Lithium, prior to ECT. Among the 29 patients receiving Valproate, the dose ranged from $600-1200 \mathrm{mg} / \mathrm{d}$, and the mean dose was $834.40 \pm 177.83 \mathrm{mg} / \mathrm{d}$. Only one patient had been treated with Lithium, with a dose of 1500 $\mathrm{mg} / \mathrm{d}$. Olanzapine, perphenazine and chlorpromazine were the most frequently administered anti-psychotics. The mean haloperidol equivalent dose among all patients was $5.31 \pm$ $4.02 \mathrm{mg} / \mathrm{d} .{ }^{20}$

The administration of physical and chemical restraints was reviewed as an indicator of improvement of illness severity. The rate of chemical and physical restraint before ECT was 77\% (30 patients) and 15.4\% (6 patients) respectively and this dropped to $7.7 \%$ (3 patients) $(\mathrm{p}<0.001)$ and $2.5 \%$ (1 patient) $(\mathrm{p}=0.031)$ after ECT administration.

The number of ECT sessions ranged from 3 to 14 with a mode of 8 sessions. None of the patients experienced severe side effects of ECT such as cognitive impairment to the degree that the physician decided to either pause or discontinue the treatment.

\section{Discussion}

The results of this study illustrate that ECT is an effective, safe, and probably underused treatment method for severe bipolar I disorder patients with a single presentation manic episode. ECT may result in a rapid decline in severity of the symptoms of mania. According to the literature, the presence of some factors such as agitation and high clinical severity are predictive of good treatment response to $\mathrm{ECT}^{21}$; and the more severe the manic symptoms are, the better the response to ECT. ${ }^{22-23}$ The most common clinical features in patients treated with ECT were psychotic symptoms (92.3\%), irritability (89.7\%), hyper-talkativeness (84.6\%) and aggression (64.1\%). Although it would have been interesting to compare these features with the clinical presentation of those manic patients who were treated pharmacologically, this data was not available in this retrospective study.

Comparison of the LOS of the ECT sample with that of patients diagnosed with a single episode of mania and exclusively treated with pharmacotherapy, raises the question as to whether the longer stays for the ECT group are a consequence of delays and hesitations in the administration of ECT in severe single manic patients responding inadequately to pharmacologic treatment. ${ }^{8}$ Based on this evidence, it might be reasonable to modify current practices which currently tend to postpone administration of ECT early in the hospitalization course, even in a single manic episode. However, it is important also to interpret our findings with caution.

In our study, it appears that delays in the application of ECT resulted in greater use of chemical restraints and the administration of intramuscular and intravenous antipsychotics. Notably, the latter are known to place the individual at increased risk for severe and life-threatening complications such as neuroleptic malignant syndrome. ${ }^{24}$ Other possible risks associated with the administration of antipsychotics to patients suffering from affective disorders (such as tardive dyskinesia) should also be taken into account. ${ }^{24}$

Moreover, the need for physical restraints (which might constitute an unpleasant memory from the time of hospitalization in patients' minds) was far greater during the period prior to ECT being administered.

Although several studies and reports regarding the application of ECT in cases of treatment-resistant bipolar disorder and refractory mania are available $e^{9,25-26}$, the literature 
seems to suffer from a paucity of information regarding such treatment early in the first-episode of the disorder. It must be noted that the present study has some limitations such as: the small number of patients: the inability to make well-designed comparisons (e.g of symptoms); and a lack of information regarding long-term outcomes of the patients reviewed in the study. Further detailed, especially prospective, studies in this area are recommended.

\section{Conclusion}

An important point is the following: rapid improvement in clinical status is likely to result in a higher level of welfare and well-being in patients experiencing their first-time admission to a psychiatry unit. This fact may play a manifest role in transforming the attitude of the public as well as patients regarding admission to psychiatry units and the use of ECT.

\section{References}

1. Weiner R. The Practice of Electroconvulsive Therapy: Recommendations for Treatment, Training, and Privileging (A Task Force Report of the American Psychiatric Association). American Psychiatric Association Committee on Electroconvulsive Therapy. 2nd ed. Washington, DC: American Psychiatric Association, 2002.

2. Abrams R. Electroconvulsive Therapy. 4th ed. Oxford: Oxford University Press, 2002

3. Barekatain M, Jahangard L, Haghighi M,Ranjkesh F. Bifrontal versus bitemporal electroconvulsive therapy in severe manic patients. Journal of ECT 2008; 24:199-202.

4. Rey JM, Walter G. Half a century of ECT use in young people. American Journal of Psychiatry 1997;54:595-602.

5. Mukherjee S, Sackeim HA, Schnur DB. Electroconvulsive therapy of acute manic episodes: a review of 50 years' experience. American Journal of Psychiatry 1994;151:169-176.

6. Devanand DP, Polanco P, Cruz $R$, et al. The efficacy of ECT in mixed affective states. Journal of ECT 2000;1 6:32-37.

7. Gruber NP, Dilsaver SC, Shoaib AM, et al. ECT in mixed affective states: a case series. Journal of ECT 2000;1 6:183-188.

8. Volpe FM, Tavares A. Impact of ECT on duration of hospitalizations for mania. Journal of ECT 2003;1 9:17-21.

9. Antunes PB, Fleck MP. Clinical outcomes and quality of life in patients submitted to electroconvulsive therapy. Journal of ECT 2009; 25(3): 182-185.

10. Lauber C, Nordt C, Falcato L, et al. Can a seizure help? The public's attitude toward electro convulsive therapy. Psychiatry Research 2005; 134:205-209

11. Walter G, Koster K, Rey JM. Electroconvulsive therapy in adolescents: experience, knowledge and attitudes of recipients. J Am Acad Child Adolesc Psychiatry 1999; 38(5): 594-599.

12. Taieb O, Flament MF, Cocos M, et al. Electroconvulsive therapy in adolescents with mood disorders: patients' and parents' attitude. Psychiatry Research 2001;104:183-190.

13. Sienaert P, Becker T, Vansteelandt $K$ et al. Patient satisfaction after electroconvulsive therapy. Journal of ECT 2005;2:227-231.

14. Goodman JA, Krahn LE, Smith GE, et al. Patient satisfaction with electroconvulsive therapy. Mayo Clinical Procedings 1999;74:967971.

15. Malekian A, Amini Z, Maracy MR, Barekatain M. Knowledge of, attitude toward, experience and satisfaction with electroconvulsive therapy in a sample of Iranian patients. Journal of ECT 2009;25:10612.

16. Ucok A, Cakir S. Electroconvulsive therapy in first- episode schizophrenia. Journal of ECT 2006;22:38-42.

17. Goodwin GM. Evidence-based guidelines for treating bipolar disorder: recommendations from the British Association for Psychopharmacology. Journal of Psychopharmacology 2003; 17:149173.

18. American Psychiatric Association. Diagnostic and Statistical Manual of Mental Disorders. 4th ed. Washington, DC: American Psychiatric Association; 1994.

19. Sackeim HA, Decina P, Prohovnik I, et al. Seizure threshold in electroconvulsive therapy: effects of sex, age, electrode placement, and number of treatments. Archives of General Psychiatry 1987;44:355-360.

20. Woods SW. Chlorpromazine equivalent doses for newer antipsychotics. Journal of Clinical Psychiatry 2003;64:663-667.

21. Small J.G., Small I.F,, Milstein V. et al. Manic symptoms: an indication for bilateral ECT. Biological Psychiatry 1985;20:1 125-1134.

22. Small J.G., Klapper M.H., Kellams J.J. et al. ECT compared with lithium in the management of manic states. Archives of General Psychiatry 1988;45:727-732.

23. Schnur D.B., Mukherjee S., Sackeim H.A., et al. Symptomatic predictors of ECT response in medication-nonresponsive manic patients. Journal of Clinical Psychiatry 1992;53:63-66.

24. Marder SR, van Kammen DP. Dopamine receptor antagonists. In: Sadock BJ, Sadock VA, eds. Kaplan and Sadock's Comprehensive Textbook of PSychiatry. 8th edition. Philadelphia, PA: Lippincott Williams \& Wilkins;2005:PP2817-2838.

25. Macedo- Soares MB, Moreno RA, Rigonatti SP, Lafer B. Efficacy of electroconvulsive therapy in treatment-resistant bipolar disorder: A Case Series. Journal of ECT 2005;21:31-34.

26. Nascimento AL, Appolinario JC, Segenreich D, Cavalcanti MT, Alves MA. Maintenance electroconvulsive therapy for recurrent refractory mania case report. Journal of Bipolar Disorders 2006;8:301-303. 